\title{
De-Centralized Multi Robot Co-Ordination and Communication
}

\author{
SANJHA KHAN*, AND BILAL ATHAR* \\ RECEIVED ON 06.09.2017 ACCEPTED ON 12.02.2018
}

ABSTRACT

The aim of this research is to develop a decentralized communication system of multiple robots which have capability to communicate and coordinate with each other, by which all robots work individually while keeping connectivity among them. Decentralized communication between robots is the main part of this project. This type of communication shows great potential in many aspects. A decentralized system is the combination of both intelligent and decision making inspired from nature. To meet this object two identical robots are developed which successfully communicate with each other to complete an assigned task. A single task is given to both robots; to find specific object/target location. Once the target is found by any of the robot that robot will then communicate with second robot to guide the path of object/target location. Guiding path to the second robot is the challenging task as discussed in this paper. The Robots use Bluetooth devices to calculate and extract the RSSI (Received Signal Strength Indication)values for communication purpose.Both robots work together as a team. By working in communicated way their time is reduced to achieve the task and create synergy. Any task could be easily attained in team work than by the work of the single individual robot.

Key Words: Multi-Robot System, Target Detection, Decentralized Coordinated System, Communication

\section{INTRODUCTION}

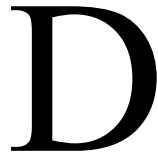
ecentralized robotic system is an effective area that has gained attention by many researchers over past few years [1-2]. This type of system gives authority to every single member to make decision on its own. There is no single centre robot that takes control for all the system. It makes the system more flexible.

Due to low cost and wide availability of robotic hardware, there is growing interest in robotics which consists of multiple simple robots instead of one highly-capable robot. In this work two swarm type robots are developed in such a way that both robots have same configuration and are designed in similar manner. Each robot has multiple sensors to detect and avoid obstacles and to reach the target location. Both robots have capability to communicate with each other by using Bluetooth. When any of the robot reaches the target first, it stops there and communicates other robot (which is still moving in the area for the search of target) to reach the same target location. 
Using single robot to find the target in any area may take more time. Single robot has to move in diverse directions of area. For working together as a team every robot may take its own specific direction, which reduced lot of time. This type of system can be used where a single robot with limited capabilities cannot perform difficult tasks (such as carrying/lifting heavy object, holding different tools for repairing purpose in industries etc.) individually.

On large scale, this type of communication algorithm has applications like: Surveillance, detecting and locating the missing object, and can be used in military and industrial operations. Swarms are ideal for such applications because a swarm of robots can spread out and effectively cover a large area in a short time. In addition, if one robot is destroyed, the rest of the robots continue to function. The control of the robot is decentralized so there is no "master" robot that controls the rest of the system; all the robots work independently to achieve the given task.

The paper is organized into five sections. Following the introduction, Section II presents the related work, Section III describes working methodology, Section IV illustrates experimental results and finally, conclusion is drawn in Section V.

\section{LITERATURE}

In literature, two obvious approaches available for motion planning of multi-robot systems are the centralized and decentralized (distributed) approaches. In centralized systems, the central or the main robot decides the path for every robot and the motion takes place after each robot is assigned its path. In the decentralized approach, there is no single coordinator in the system. Each agent coordinates its own movement and ensures that it does not collide with any obstacle or any other robot during goal accomplishment [3].
Several centralized approaches for robot navigation are discussed in [4-7]. One other approach is 'Multi Robot Communication and Target Tracking System with Controller Design and Implementation of SWARM Robot Using Arduino'. In this work the authors present hardware specifications and controller design of robot for swarm applications. Implementation details are explained and applications of the multi-agent system are verified through algorithms. Two different algorithms are implemented for the communication of multi-robots. Leader-Follower Approach and Move to Goal Approach [8]. Although both the algorithms are simple and efficient but the methodologies used are completely centralized, motion of one robot to reach the target completely depends on the other robot.

Decentralized approach offers several advantages over centralized algorithms including flexibility, robustness, intelligent decision making etc. Over last two decades the decentralized robotic approach has achieved a significant progress. This type of approach appears in the field of artificial swarm intelligence as well as biological studies of insects and other fields in nature.

The work presented in 'Artificial potential field controllers for robust communications in a network of swarm robots' [9] includes many simple robots working together to perform tasks which are beyond the capability of single robot acting alone. In order to work together effectively they maintain a reliable wireless communication network among themselves. For performing the complete task an overhead camera is used in working field, which captures the snapshot of the scene. The image is sent to base station $\mathrm{PC}$ and processed using MATLAB. The orientation, position and coordination of vertices of obstacle are extracted from the processed picture. So these robots are capable to work in the area which is already

Mehran University Research Journal of Engineering \& Technology, Volume 38, No. 1, January, 2019 [p-ISSN: 0254-7821, e-ISSN: 2413-7219] 
known where camera is fixed. Sheludko et. al. [10] present a group of autonomous robots that uses fuzzy logic for the robot movement, obstacle avoidance and achieving goals. The robot moves via the signals from the remote workstations and receive the signals from the technical vision based control system.

Coordination and communication are two challenging tasks. For solving the coordination problem significant work has been done. Amato et. al. [11] introduce a method for multi robot coordination. Multiple waiter robots are designed that take drinks from bartender and serve it to the customers. Avola et. al. [12] presents the prototype of an autonomous robot which uses two algorithms, SLAM (Simultaneous Mapping and Localization) for mapping unknown areas and other algorithm for object detection. The aim of this paper is to present an autonomous robot system which can detect the target object without colliding any obstacle in an unknown environment. The robot works in a manner that it develops map from the acquired data of ultrasonic sensor as it moves in the environment. And it also looks for the target object while avoiding the disturbing obstacles in the path.

Jahn et. al. [13] present a distributed approach where the team of robots moves around the specific area in order to avoid internal or external agents crossing through the delimited area. Authors split problem in two parts. First, presents distributed planning method for boundary. Second, all robots maintain equal distance and communicate only with neighbor robot.

Taking inspiration from insects and ant colony behavior number of swarm algorithms are designed, where collection of swarm has potential to perform task collectively. These algorithms require communication between robots to allow the robots to coordinate their behavior and act collectively. In Hoff et. al. [14] similar concept is used where robot acts as stationary environment beacon or a wandering robot. The two algorithms (virtual pheromone and cardinality) are used to construct path between food and nest. Both algorithms provide effective method of coordination but one has to sacrifice some robots to act as beacon to hold values.

In our work we present improved performance over previous methods. The methodology used in this work makes the system completely decentralized. Including this once the robots are programmed they are capable to work in any new field without any human help.

\section{METHODOLOGY}

Two robots have been developed in such a way that both robots have same configuration and are designed in similar manner to achieve the specific task. The Robots are composed of a Controller [15], three-wheeled Robot chassis [16], Ultrasonic Sensor [17], Color Sensor [18] and Bluetooth [19]. Fig. 1 shows the block diagram of basic architecture of robot. Fig. 2 shows the final design of the robot.

The task has been accomplished in two steps.

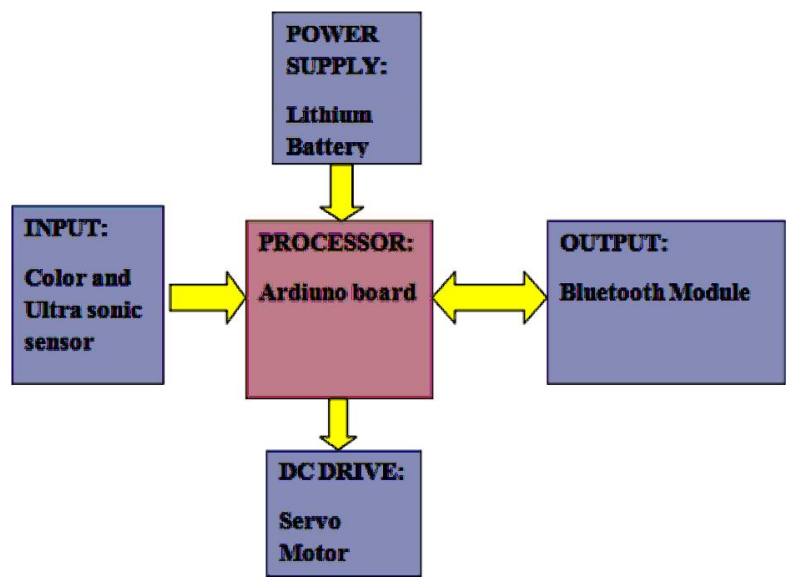

FIG. 1. BLOCK DIAGRAM OF ROBOT

Mehran University Research Journal of Engineering \& Technology, Volume 38, No. 1, January, 2019 [p-ISSN: 0254-7821, e-ISSN: 2413-7219] 
To Reach Target Location: As the power is turned on, the robot moves in forward direction. It continues moving in same direction until any obstacle appears in the path at $20 \mathrm{~cm}$ distance. If the sensor detects an obstacle in the range the robot stops and turns left to measure left side distance, then turns right to measure right side distance. It compares both measured distances if the left distance value is greater than right distance value, the robot moves left else it moves right. It repeats this process whenever sensor detects any obstacle in specified range.

To differentiate between obstacles and target certain parameters (like size, height or color of object) needs to be specified. For this work the color of object is chosen to differentiate between obstacles and target. To detect the specific color, the color coding is developed. The color sensor has capability to identify the strength of RGB (Red, Green and Blue) colors contained in the object. The strength of each color is specified by range from 0 255. For this task the color of target is set as RED color. If the color strength matches the strength of red color the robot stops there. Otherwise the robot considers object as obstacle and takes left or right direction from the obstacle.

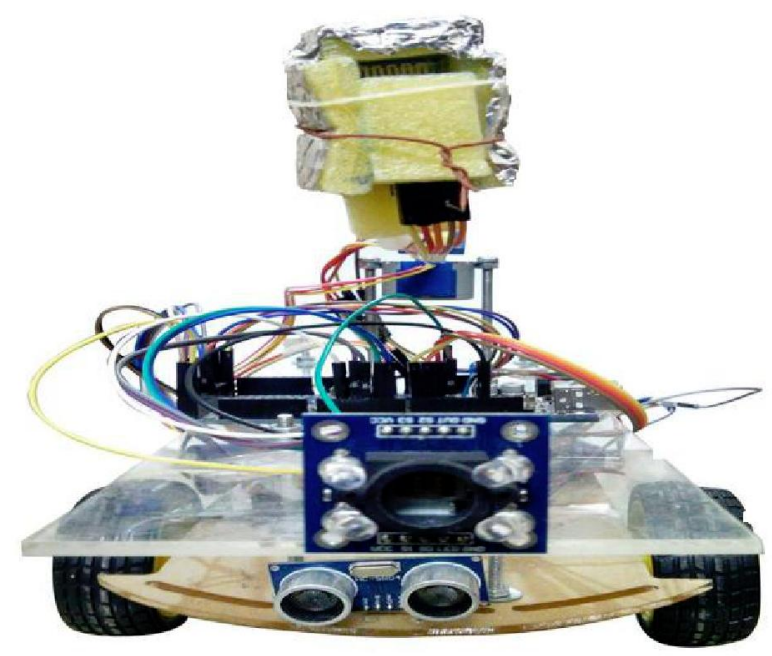

FIG. 2. FINAL DESIGN OF ROBOT EQUIPPED WITH MULTIPLE SENSORS AND BLUETOOTH AT THE TOP
Both robots adopt the same mechanism to achieve the target. Any of robot reaches the target first, it communicates the other (which is still moving in the area for the search of target) to reach the same place immediately.

Communicate with Other Robot: Bluetooth are the devices can be used for communication between two robots. It can be used in a Master or Slave configuration. In this work initially both the Bluetooth devices are simply in "ON" position. But the device which first reaches the target acts as a master device and sends signal to other Bluetooth device, while the other device acts as a slave. The slave device extracts and calculates the RSSI values to measure the signal intensity in all directions. The robot decodes RSSI values and advances in the direction where the signal strength is greatest. By tracing the signal intensity, the robot reaches the master robot at target location.

According to the algorithm, if any obstacle appears in the path while moving towards master robot. The slave robot repeats the above mentioned methodology to differentiate obstacle and target. If it is obstacle it will take left or right direction and rotate its Bluetooth to get largest RSSI values and move towards master robot. It repeats the same for every obstacle before reaching the master robot. Fig. 3 shows the complete algorithm developed for achieving task.

\section{RESULTS}

The complete robotic system was tested in specified area to materialize the decentralized communication system of multi robots. Fig. 4(a-f) show the snapshots of experimental results to reach the target by two robots. The sequence of operations is as follows: In 


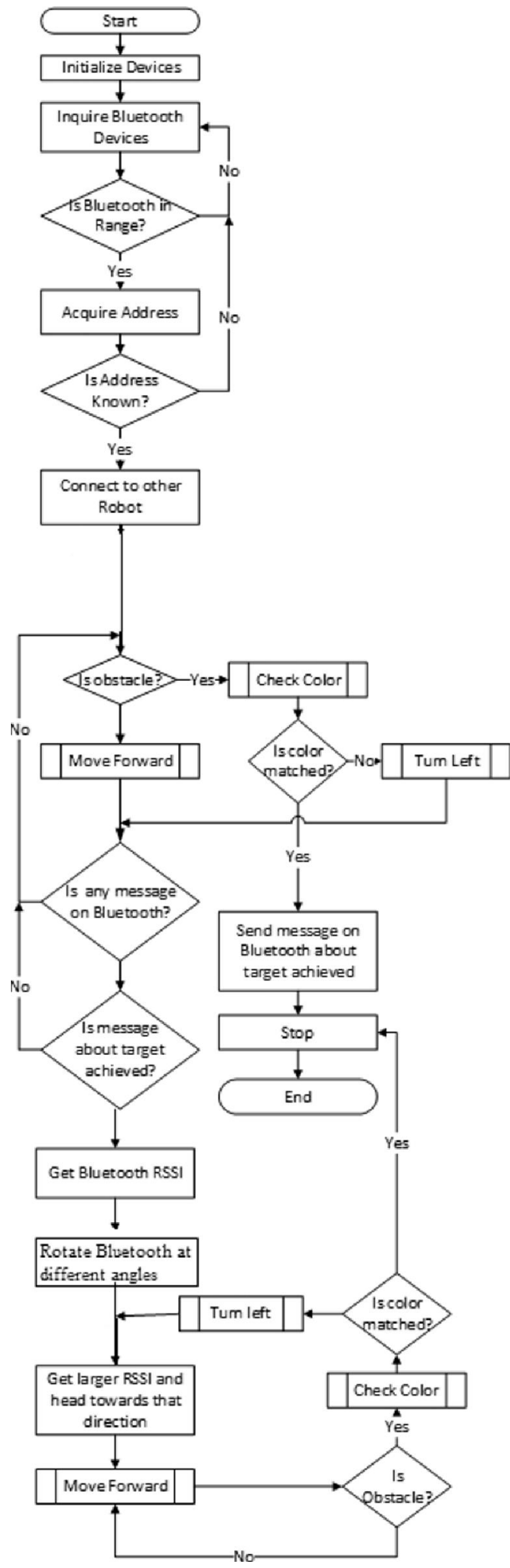

FIG. 3. FLOWCHART SHOWING WORKING ALGORITHM OF BOTH ROBOTS
Fig. 4(a) two identical robots start moving to search the target object. In Fig. 4(b) Robot1 moving randomly faces the obstacle (white colored box) in the path, while Robot 2 continues to move in different directions. Fig. 4(c) shows that Robot1 pauses there pivot left and measures distance and Fig. 4(d) shows that Robot1 pivots right, measures distance. By comparing both distances in Fig. 4(e) the robotl advances towards right. In Fig. 4(f) Robot2 reaches the target object, while Robot1 keeps on moving.

After reaching the target object, Robot2 sends signal via Bluetooth to guide robot1 about the targeted location. Fig 5 shows further communication steps: In Fig. 5(a) Robot1 receives signal from robot2. It uses RSSI to measure signal intensity in different directions. In Fig. 5(b-d) Bluetooth devices rotate at different angles to search for highest signal intensity. In Fig. 5(e) Robot1 EN routes the direction where signal intensity is the greatest. In Fig. 5(f) Robot 1 reaches the target object.

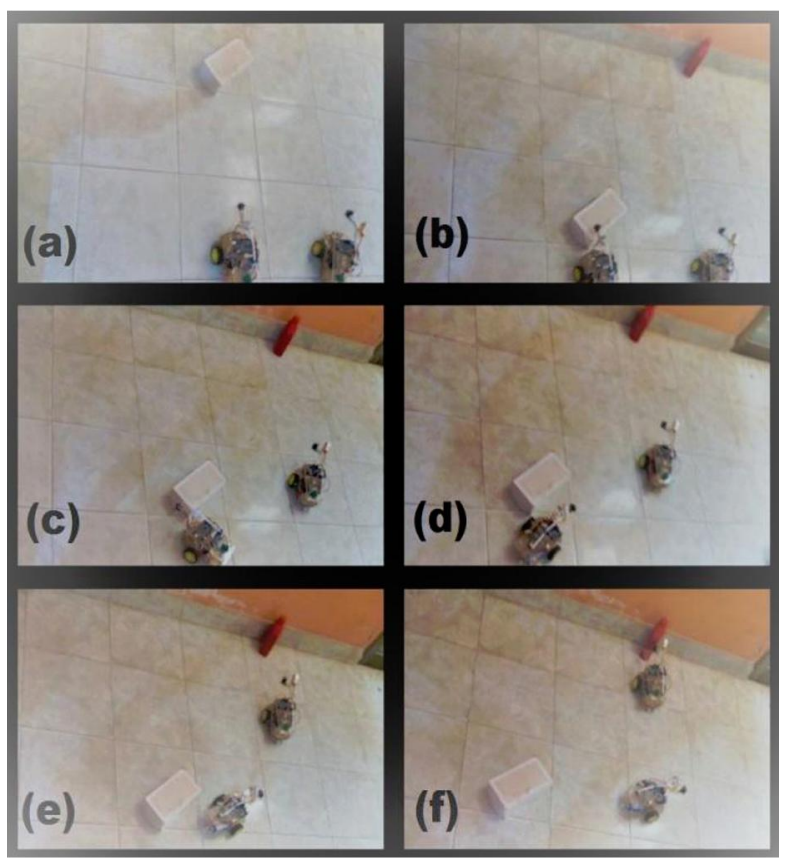

FIG. 4. EXPERIMENTAL RESULTS TO REACH THE TARGET

Mehran University Research Journal of Engineering \& Technology, Volume 38, No. 1, January, 2019 [p-ISSN: 0254-7821, e-ISSN: 2413-7219] 
During seeking target both robots work individually. They neither depend on each other nor on any other central controlling system. But as one of them reached target it took decision to act as master. This type of coordination and communication make the system decentralized.

Fig. 6 shows snapshots of trials taken in a closed area of $8 \times 9 \mathrm{ft}$ with different obstacles and by placing target object at 3 different locations.

To measure the effectiveness of team work the experiment was performed with two robots and single robot

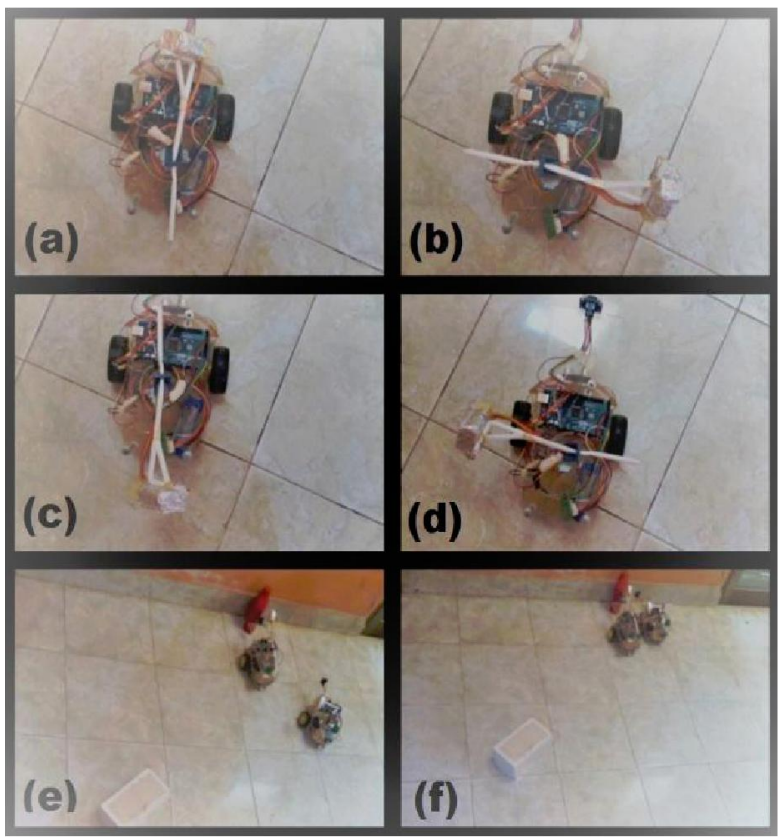

FIG. 5. EXPERIMENTAL RESULTS OF COMMUNICATION PROCESS individually. Different number of obstacles were placed in the specified area. Four trials were taken with each number of obstacles by single and by two Robots. Table 1 shows the average time taken by single and two robots to reach the target.

With the completion of this project it is experienced that the robots performed the assigned tasks by communicating and making decisions between them without any human guidance. In addition, it is also observed that two Robots working together as a team take less time as compared to single Robot doing the same task alone.

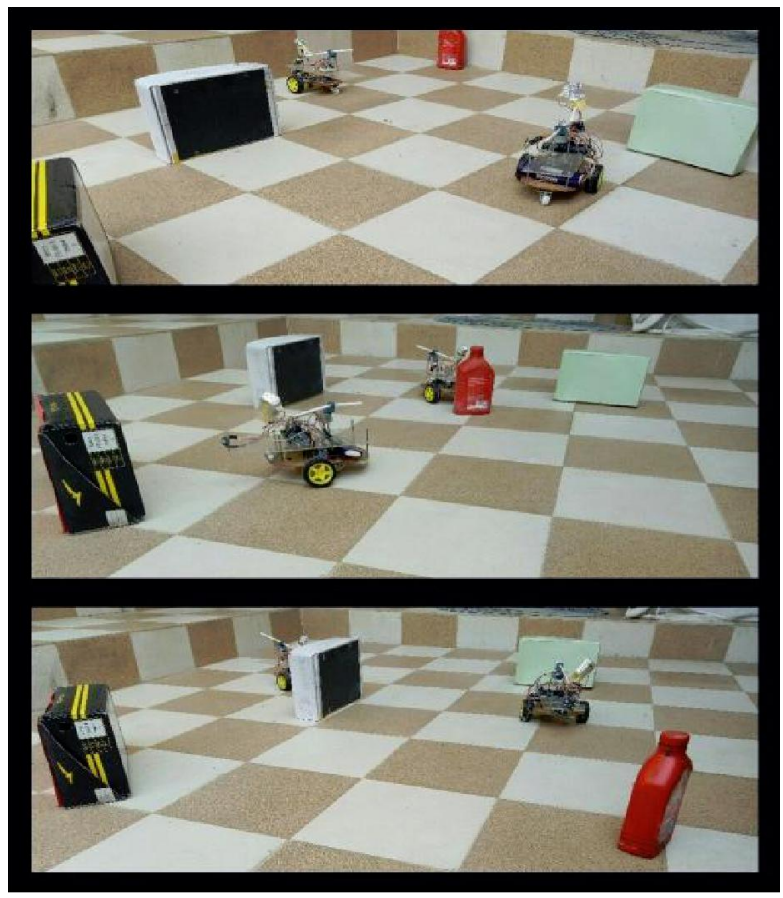

FIG. 6. MULTIPLE SCENARIOS WITH DIFFERENT TARGET POSITION

TABLE 1. TIME TO COMPLETE TASK BY SINGLE VS TWO ROBOTS

\begin{tabular}{|c|c|c|c|c|}
\hline No. of Robots & No. of Obstacles & Average Time(s) & Difference(s) & Area \\
\hline Single & \multirow{2}{*}{1} & 57 & \multirow{2}{*}{18} & \multirow{6}{*}{$8 \times 9$ sq.f } \\
\hline Two & & 39 & & \\
\hline Single & \multirow{2}{*}{2} & 80 & \multirow{2}{*}{17} & \\
\hline Two & & 63 & & \\
\hline Single & \multirow{2}{*}{3} & 116 & \multirow{2}{*}{23} & \\
\hline Two & & 93 & & \\
\hline
\end{tabular}

Mehran University Research Journal of Engineering \& Technology, Volume 38, No. 1, January, 2019 [p-ISSN: 0254-7821, e-ISSN: 2413-7219] 


\section{CONCLUSION}

Two robots were assigned a task to find a target with certain parameters. Color sensors were used for this purpose. To make system efficient enough ultrasonic sensors were installed to avoid the collision. When one robot reached the target object it then successfully communicated second robot by using Bluetooth device and guided its location by transmitting signals. The second robot calculated the RSSI values and successfully traveled in a direction where there is greatest signal strength value.

\section{FUTURE WORK}

Although this project successfully completed the assigned task and worked properly, but still there are some limitations and areas that can be looked and reviewed for further improvements.

Firstly, Communication algorithm needs more improvement. During communication if any obstacle appears in the path, the slave robot may not be able to reach the master robot directly. Secondly for wide area and greater ranges Bluetooth device and color sensor could be replaced with other communication device and high definition camera.

\section{ACKNOWLEDGEMENT}

The authors are heartily thankful to Prof. Dr. Bhawani Shankar Chowdhry, Dean, Faculty of Electrical, Electronic \& Computer Engineering, Mehran University of Engineering \& Technology, Jamshoro, Pakistan, for his cooperation and supervision for acquiring this task. The authors would also like to acknowledge Engr. Azam Rafique Memon, for his cooperation, supervision and encouragement. He made it possible for us to complete the project.

\section{REFERENCES}

[1] Muthusamy, R., Bechlioulis, C.P., Kostas, J.K., and Kyrki, V., "Task Speciûc Cooperative Grasp Planning for Decentralized Multi-Robot Systems", IEEE International Conference on Robotics and Automation, pp. 6066-6073, Seattle, Washington, USA, 2015.

[2] Wiktor, A., Scobee, D., Messenger, S., and Clark, C., "Decentralized and Complete Multi Robot Planning in Confined Spaces", IEEE International Conference on Intelligent Robots and System, pp. 1168-1175, Chicago, IL, USA, 2014.

[3] Doriya, R., Mishra, S., and Gupta, S., "A Brief Survey and Analysis of Multi-Robot Communication and Coordination", International Conference on Computing, Communication and Automation, pp. 1041-1021, Noida, India, 2015

[4] Carpin, S., and Pagello, E., "On Parallel RRTS for MultiRobot Systems", 8th Conference Italian Association for Artiûcial Intelligence, pp. 834-841, 2002.

[5] Luna, R., and Bekris, K.E., "Efficient and Complete Centralized Multi Robot Path Planning", IEEE International Conference on Intelligent Robots and Systems, pp. 201-202, San Francisco, CA, USA, 2011.

[6] Luna, R., and Bekris, K.E., "Push and SWAP: Fast Cooperative Path Finding with Completeness Guarantees", International Joint Conference on Artiûcial Intelligence, pp. 294-300, Barcelona, Catalonia, Spain, 2011 .

[7] Peasgood, M., Clark, C.M., and McPhee, J., "A Complete and Scalable Strategy for Coordinating Multiple Robots within Roadmaps", IEEE Transactions on Robotics, Volume 24, No. 2, pp. 283-292, Piscataway, NJ, USA, 2008.

[8] Patil, D.A., Upadhye, M.Y., Kazi, F.S., and Singh, M.N., "Multi Robot Communication and Target Tracking System with Controller Design and Implementation of SWARM Robots Using Arduino", International Conference on Industrial Instrumentation and Control, pp. 412-416, Pune, India, 2015. 
[9] Dunbar, T.W., and Esposito, J.M., "Artiûcial Potential Field Controllers for Robust Communications in a Network of SWARM Robots", Proceedings of Annual Southeastern Symposium on System Theory, Volume 37, pp. 401-405, Tuskegee, AL, USA, 2005.

[10] Sheludko, V.N., Putov, V.V., Ignatiev, K.V., Kopichev, M.M., and Serykh, E.V., "Decentralized Control of Cooperative Robotic System", IEEE 5th Forum Strategic Partnership of Universities and Enterprises of Hi-Tech Branches, pp. 15-18, Petersburg, Russia, 2016.

[11] Amato, C., Konidaris, G., Anders A., Cruz, G., How, J.P., and Kaelbling, L.P., "Policy Search for Multi-Robot Coordination under Uncertainty”, International Journal of Robotics Research, Volume 35, No. 14, pp. 1760-1778, Thousand Oaks, CA, USA, 2016.

[12] Avola, D., Foresti, G.L., Cinque, L., Massaroni, C., Vitale, G., and Lombardi, L., "A Multipurpose Autonomous Robot for Target Recognition in Unknown Environment”, IEEE $14^{\text {th }}$ International Conference on Industrial Informatics, pp. 766-771, Poitiers, France, 2016.

[13] Jahn, A., Alitappeh, R.J., Salda na, D., Pimenta, L.C.A., Santos, A.G., Mario F.M., and Campos, M.F.M., "Distributed Multi-Robot Coordination for Dynamic Perimeter Surveillance in Uncertain Environments", IEEE International Conference on Robotics and Automation, pp. 273-278, Singapore, 2017.
[14] Hoff, N.R., Sagoff, A., Wood, R.J., and Nagpal, R., “Two Foraging Algorithms for Robot SWARMS Using Only Local Communication", International Conference on Robotics and Biomimetics, pp. 123-130, Tianjin, China. 2010 .

[15] "Arduino mega2560 r3 (High Quality) a0004 electronics wall”, http://www.ewallpk.com/index.php?id product $=2 \&$ controller $=$ product, $\left(\right.$ Visited on $2^{\text {nd }} \mathrm{July}$, 2016).

[16] "2WD 3 Wheel Robotic Smart Car Chassis a0080 Electronics Wall,” http://www.ewallpk.com/index.php?id product $=172 \&$ controller $=$ product $\&$ search query $=$ robot $\&$ results $=31$, (Visited on $2^{\text {nd }}$ July, 2016).

[17] "HC-SR04 Ultrasonic Sensor Module a0001 - Electronics Wall”, http://www.ewallpk.com/index.php?id product $=69 \&$ controller $=$ product $\&$ search query $=$ ultrasonic $\&$ results $=4$, (Visited on $2^{\text {nd }}$ July, 2016).

[18] “TCS3200 Color Sensor Module a0401 - Electronics Wall”, http://www.ewallpk.com/index.php?id product $=360 \&$ controller $=$ product $\&$ search query $=$ color + sensor \&results=13, (Visited on $2^{\text {nd }}$ July, 2016).

[19] "HC-05 Bluetooth RF Transceiver Module (Master/ Slave) a0409 Electronics Wall”, http://www.ewallpk.com/ index.php? id product $=368 \&$ controller $=$ product $\&$ search query=bluetooth\&results $=16$, (Visited on $2^{\text {nd }} \mathrm{July}, 2016$ ). 